\title{
Identification of Near-Field and Far-Field Using Confocal Microscopy Setup for Optical Sensing Applications
}

\author{
W.M. Mukhtar ${ }^{1,2^{*}}$, N.Z.M. Zulkifli ${ }^{1}$ and R.M. Halim³ \\ ${ }^{1}$ Applied Physics Programme, Faculty of Science and Technology, \\ Universiti Sains Islam Malaysia (USIM), 71800 Negeri Sembilan, Malaysia \\ ${ }^{2}$ Frontier Materials Research Group (FMRG), Faculty of Science and Technology, \\ Universiti Sains Islam Malaysia (USIM), 71800 Negeri Sembilan, Malaysia \\ ${ }^{3}$ National Metrology Institute of Malaysia (NMIM), SIRIM Berhad, \\ Bandar Baru Salak Tinggi, 43900 Sepang, Selangor, Malaysia
}

\begin{abstract}
The presence of near-field indicates the existence of evanescent waves, which is one of the important requirements for the development of an optical sensor. This study was carried out to identify the presence of near-field and far-field as monochromatic light propagated through various structures and sizes of apertures. The confocal microscopy setup consisted of He-Ne laser with $633 \mathrm{~nm}$ of excitation wavelength, apertures (slits and numerous sizes of pinholes), objective lens, projected screen and silicon photodetector. The near-field and far-field were determined by calculating the Fresnel number, $F_{N}$ as assorted size and design of apertures were added in the setup. The presence of far-field was identified with the usage of slits (single slits and double slits) represented by the value of $\mathrm{F}_{\mathrm{N}}$, which was less than $1\left(\mathrm{~F}_{\mathrm{N}}<<1\right)$. As the aperture was replaced with pinholes with diameters within $30 \mu \mathrm{m}$ and $1.2 \mathrm{~mm}$, the near-field was resulted $\left(\mathrm{F}_{N}>>1\right)$. Value of $F_{N}$ became greater as distance $\mathrm{x}$ between lens and pinholes increased. We also discovered a significant finding where the field intensities $I$ were decreased with increment of distance $\mathrm{x}$ for the near-field, and vice versa (far-field). By using an extrapolating technique, it was found that the near-field can be created by using pinhole with a diameter of $\mathrm{a}<1.46 \mathrm{~mm}$. In conclusion, the discovery of this work proves an excellence role of pinholes in confocal microscopy setup in creating the evanescent waves for optical sensing applications.
\end{abstract}

Keywords: near-field; far-field; pinholes; Fresnel number; evanescent wave

\section{INTRODUCTION}

Scanning near-field optical microscopy (SNOM or NSOM) is at the spearhead of today's technology due to its impressive potential in nanoscanning applications (Kawata et al., 2001; Szymanski et al., 2005). This microscopy technique breaks the far-field resolution limit by utilizing the characteristics of evanescent waves. In SNOM, the excitation laser light is focused through an aperture with a diameter smaller than the excitation wavelength, resulting in an evanescent field (or near-field) on the far side of the aperture. The ability of nanoscanning mainly influenced by the probe's structure such as conical taper dimension and surface roughness, types of coating materials and fabrication techniques (Mukhtar et al., 2012; Zhang et al., 2016; Urrutia et al., 2016; Kharat et al., 2006; Huo et al., 2006). In far-field optical microscopy, the object is illuminated by a monochromatic plane wave. The transmitted or reflected light, scattered by the object in a characteristic way, is collected by a lens and imaged onto a detector (Xie et al., 2014). Diverse types of applications in SNOM have been developed such as far-field illumination 
and far-field detection, near-field illumination and far-field detection, far-field illumination and near-field detection and near-field illumination and near-field detection (Novotny \& Hecht 2006).

To make these applications successful, optical fibre probes are fabricated using several types of techniques. Few common fabrication techniques of probes are heatingand-pulling (Halip et al., 2016; Harun et al., 2013; Mukhtar et al., 2010; Musa et al., 2016), chemical etching (Cao et al., 2017; Chen \& Shen 2016) and combination of heating-and-pulling and chemical etching (Mukhtar et al., 2012a; Chen et al., 2014). Chemical etching is a very interesting method because it can produce a considerable number of identical tips for batch fabrication (Hecht et al., 2000). The main drawback of this method is the production of rough surface on the tapered part. The heatand-pull technique results in a smooth conical tapered surface (Mukhtar et al., 2012b). Unfortunately, this technique enables to produce identical tips since the fabrication process is limited to one fibre for each run. Among these techniques, the combination method is more favourable due to its potential in fabricating identical tips and smooth tapered area. To avoid light propagation loss from fibre tapered area to the environment, metal thin film such as aluminium, silver and gold are coated on the conical taper area by using thermal evaporation, electronbeam (e-beam) evaporation or by sputtering technique (Namenko et al., 2014; Tao et al., 2016; Yu et al., 2016).

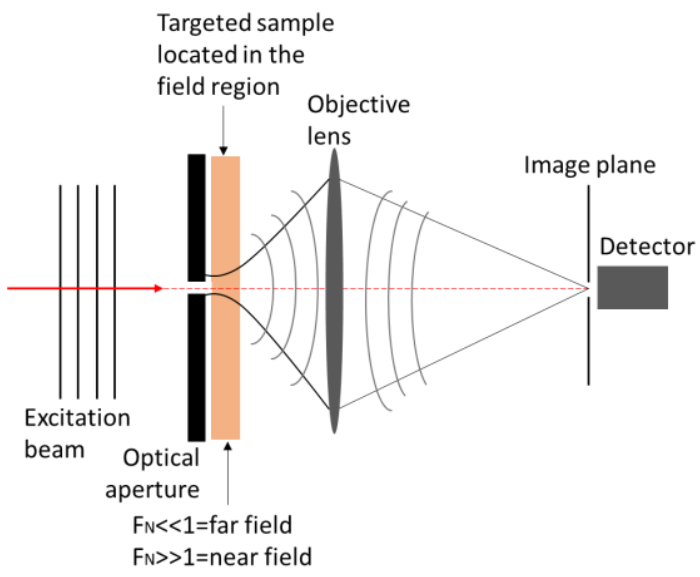

Figure 1. Optical microscopy viewed as a generalisation of confocal microscopy. The presence of near-field indicates the existence of evanescent waves which can be used for sensing application in detecting the targeted sample (Novotny \& Hecht 2006)
Besides the application of optical fibre probe, the nearfield can also be created by employing a confocal microscopy setup which is less costly and much simpler than probe's fabrication. Confocal microscopy is a technique that is applied in the scientific discipline by irradiates the targeted sample with focused light originating from a source and directs the response from the sample onto a pinhole (Novotny \& Hecht 2006). Light from a laser source is spatially filtered by sending it through an aperture. After propagating through the aperture, light is collimated by a lens, as shown in Figure 1. Size of aperture plays a significant role in yielding the near-field or far-field. The existence of near-field indicates the presence of evanescent waves which is one of the important criteria in optical sensing (Eleftheriades et al., 2008; Maliakal et al., 2016; Ton et al., 2015). As the targeted sample or analyte is located in the near-field region, the detector will detect the change in refractive index sensing properties of the optical system.

This study was carried out to identify the presence of near-field and far-field for future applications in optical sensing. Firstly, the diffraction pattern from various size and structures of apertures such as slits and pinhole were observed. Next, the properties of beam spot size, the distance between each fringe and the intensity of field were investigated. The identification of near-field and far-field relied on the value of Fresnel number, $F_{N}$. The main finding of this work indicates the potential application of pinhole in confocal microscopy to generate an evanescent wave in the near-field region for sensing application. The output of this study can be used as an indicator to estimate the appropriate diameter size of fibre tips for detection purpose based on pinhole's size. We believe that this discovery will contribute to the development of the less costly and high sensitive optical sensor.

\section{METHODS}

A helium-neon laser (Brand: LASOS) with an excitation wavelength of $633 \mathrm{~nm}$ and a maximum power of $1 \mathrm{~mW}$ was used as a light source. The optical apertures such as single slits, double slits and assorted sizes of pinholes $(30 \mu \mathrm{m}$, o.8mm, 1.0mm and 1.2mm, (Brand: Edmund Optic)) were positioned between laser and lens. The distance between pinhole and lens, $x$ was varied between $x=5 \mathrm{~cm}$ and $x=50 \mathrm{~cm}$ with an increment of $5 \mathrm{~cm}$ for each measurement. For light 
collimation purpose, two types of the objective lens with a focal length of $f=20 \mathrm{~mm}$ and $f=100 \mathrm{~mm}$ were located on the rail bench between pinhole and screen. The images represented by diffraction patterns (fringes) appeared on the white screen. Based on that observation, the distance between one fringe to another fringe was determined.

To study the standard properties of light propagation such as field intensity and a number of fringes, the experiment was started without the presence of apertures. The fringes image on the projected screen was captured by using a digital camera. Next, the apertures were inserted between the laser and lens to observe the diffraction patterns. The diffraction images, which consisted of a series of fringes, were analysed. The power intensity of the image was detected and recorded by using a silicon photodetector and optical power meter, respectively (Brand: Newport).

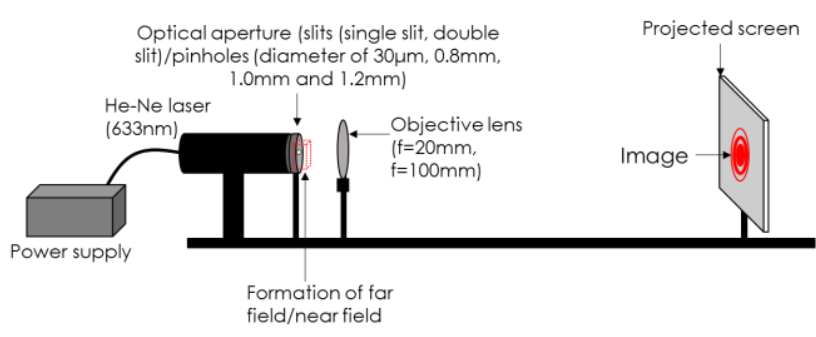

Figure 2. Experimental setup for determination of nearfield and far-field regions through numerous sizes of optical apertures

When an optical wave is transmitted through an aperture on an opaque screen, the diffraction patterns represented by intensities distributions were observed. The diffraction pattern might change significantly from the aperture shadow depending on the distance between the aperture and observation plane, the wavelength and the dimensions of the aperture. There are two types of diffraction pattern which are known as Fraunhofer diffraction (far-field) and Fresnel diffraction (near-field). The presence of the nearfield and the far-field was determined by calculating the value $\mathrm{F}_{\mathrm{N}}$ as expressed in Equation 1;

$$
F_{N}=A / \lambda z
$$

Where;

$\mathrm{A}=$ an area of circular image with $\mathrm{r}$ is the radius of the circular image

$\lambda=633 \mathrm{~nm}$ represents light excitation wavelength

$\mathrm{z}=$ the distance between aperture and screen

If $\mathrm{F}_{\mathrm{N}}>>1$, the regions can be classified as near-field region. The far-field approximation is valid if the propagation distance between the aperture and observation planes is large, which results in $\mathrm{F}_{\mathrm{N}}<<1[28,29]$. To determine the maximum size of aperture for the presence of the near-field, a graph extrapolation technique was performed.

\section{RESULTS AND DISCUSSION}

Figure 3(a)(i) illustrates the formation of fringes as the light was collimated using an objective lens with focal length, $\mathrm{f}=\mathbf{2 0} \mathrm{mm}$ without the presence of optical aperture. The formation of circular rings image with a diameter of beam size $d=1.2 \mathrm{~cm}$; consisted of ten sets of fringes were observed on the projected screen. The field intensities were decreased from $\mathrm{I}=7.95 \mathrm{~W} / \mathrm{m}^{2}$ to $\mathrm{I}=5.5131 \mathrm{~W} / \mathrm{m}^{2}$ when the distance between laser and lens increased. As the focal length was increased at $\mathrm{f}=100 \mathrm{~mm}$, the number of fringes was reduced to four fringes, which results in a smaller beam spot size with a diameter of $\mathrm{d}=0.6 \mathrm{~cm}$ as depicted in Figure 3(a)(ii). The distance between each fringe was obtained between $0.0128 \mathrm{~mm}$ and $0.0057 \mathrm{~mm}$. The field intensities show the same behaviour with $f=100 m m$ where the values were decreased with the increment of the distance between laser and lens. However, in the comparison between $\mathrm{f}=\mathbf{2 0 \mathrm { cm }}$ and $f=100 \mathrm{~cm}$, the values of field intensities using $f=\mathbf{2 0} \mathrm{cm}$ were smaller than $\mathrm{f}=100 \mathrm{~cm}$. Diameters of beam spot became smaller as the optical aperture was located between the laser and lens due to the spatial filtering factor (Saleh \& Teich 1991).

Figure $3(\mathrm{~b})(\mathrm{i})$ portrays the image of fringes as a single slit was added in the experimental setup with $f=20 \mathrm{~cm}$. The diameter of the beam spot size was increased by about $3.33 \%$ as the single slit was inserted. The average size of the beam spot was obtained as $d=1.24 \mathrm{~cm}$. Ten fringes were captured on the screen as the distance between aperture and lens was 
set at $x=5 \mathrm{~cm}$. A minimum number of six fringes were observed as the value of $x$ was increased until $50 \mathrm{~cm}$. The formation of a smaller beam spot size was observed with an average diameter of $d=0.39 \mathrm{~cm}$ as the focal length was increased at $f=100 \mathrm{~cm}$ (Figure $3(\mathrm{~b})(\mathrm{ii})$ ). The number of fringes remains the same as $f=\mathbf{2 0} \mathrm{cm}$.

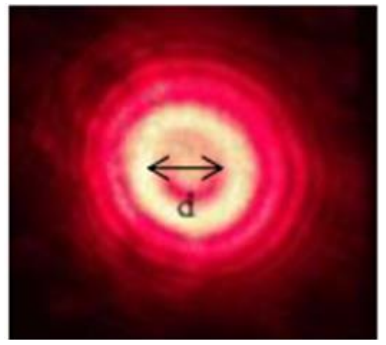

(i)

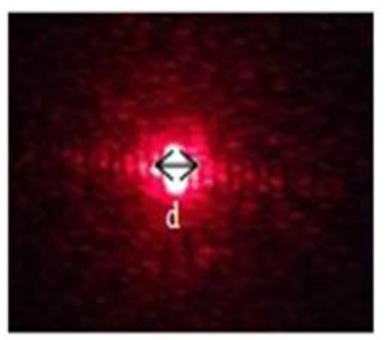

(i)

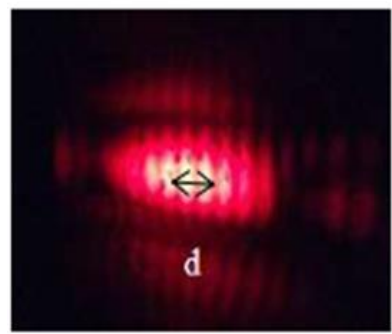

(i)

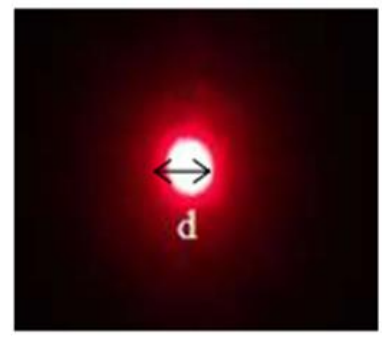

(ii)

(a)

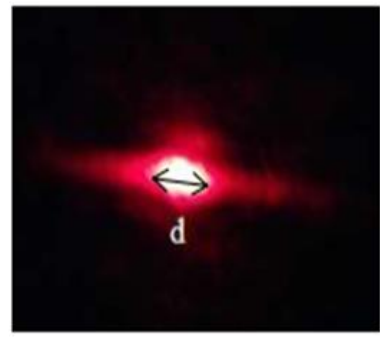

(ii)

(b)

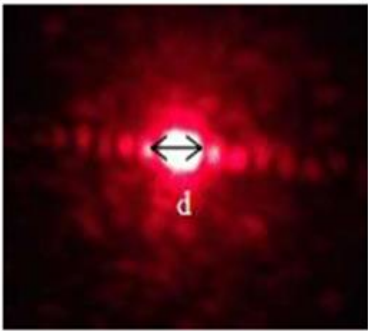

(ii) (c)

Figure 3. Formation of fringes on screen a convex lens with (i) $f=20 \mathrm{~mm}$ (ii) $f=100 \mathrm{~cm}$ using different types of apertures; (a) without slit aperture; (b) single slit apertures; (c) double slits apertures

The appointment of double slit aperture resulted in an enhancement of beam spot size to $d=1.35 \mathrm{~cm}$ (about $4.17 \%$ in comparison without the presence of slit) as the focal length was set at $f=20 \mathrm{~cm}$. The number of fringes was decreased in which around eight to six fringes were captured on the projected screen as distance $x$ between the lens, and the double slit was modulated as shown in Figure 3(c)(i). When $f=100 \mathrm{~cm}$ (as illustrated in Figure 3(c)(ii)), an equivalent size of beam spot with Figure 3(b)(ii) was resulted with $\mathrm{d}=0.39 \mathrm{~cm}$. The number of fringes also indicated a similar pattern with Figure 3(c)(i).

Next, the experiment was carried out by replacing the slits aperture with numerous sizes of pinhole apertures, as illustrated in Figure 4. The properties of images which formed on the screen such as a number of fringes and beam spot size were observed. Since part of the light was filtered as it entered the pinhole, the image produced was smaller than without the presence of pinhole. Figure 4(a) shows the images of circular rings as light propagated through a pinhole with a diameter of $\mathrm{a}=1.2 \mathrm{~mm}$. The diameter of the beam spot was obtained as $\mathrm{d}=0.0129 \mathrm{~mm}$. At $\mathrm{x}=5 \mathrm{~cm}$, ten sets of fringes were captured on the screen. As x was increased until 50cm, the number of fringes was reduced to six. The number of fringes on the screen decreased from nine to five fringes as the diameter of a pinhole was reduced to $a=1.0 m m$ as depicted in Figure 4(b). The beam spot size was obtained as $d=0.0154 \mathrm{~mm}$. The diameter of the beam spot was increased to $d=0.0193 \mathrm{~mm}$ with a number of fringes within eight to four were obtained as the pinhole size was reduced to $\mathrm{a}=0.8 \mathrm{~mm}$ (Figure $4(\mathrm{c})$ ). Figure $4(\mathrm{~d})$ portrays an unclear image of diffraction pattern as light propagated through the pinhole aperture with a diameter of $\mathrm{a}=30 \mu \mathrm{m}$. Due to the indefinite image which we believed was caused by the presence of the strong near-field, further analysis was unable to be performed using $30 \mu \mathrm{m}$ pinhole.

The existence of near-field and far-field can be determined by calculating the Fresnel number, $F_{N}$ (Mukhtar et al., 2012c). If $F_{N}<<1$, the field can be classified as far-field, meanwhile if $F_{N}>>1$, the near-field can be said as existing within that respective region. The presence of near-field indicates the appearance of the evanescent field (Kawata 2001). Figure 5 illustrates the values of $F_{N}$ for single slit and double. 


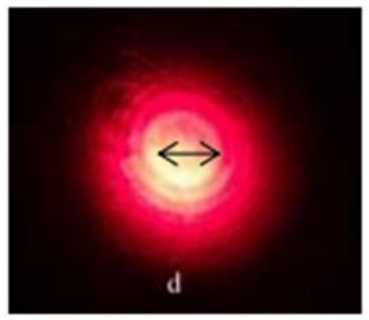

(a)

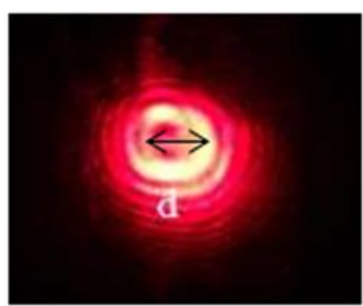

(c)

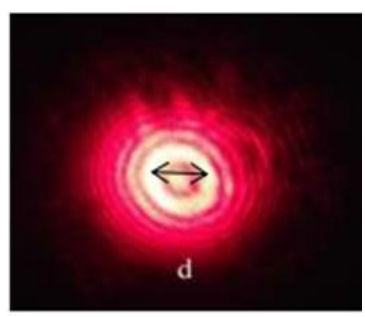

(b)

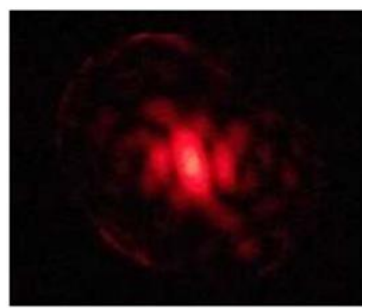

(d)
Figure 4. Formation of fringes through a convex lens with $\mathrm{f}=20 \mathrm{~cm}$ using various diameters of pinhole, a (a)1.2 $\mathrm{mm}$ (b) $1.0 \mathrm{~mm}$ (c) $0.8 \mathrm{~mm}$ (d) $30 \mu \mathrm{m}$

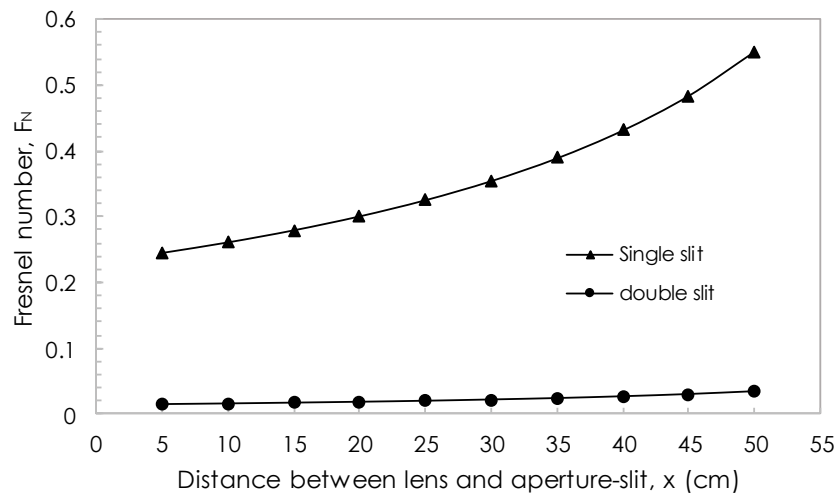

Figure 5. The presence of far-field $\left(F_{N}<<1\right)$ as light propagated through slit apertures as the distance between lens and slits were increased. The values of $F_{N}$ for both were smaller than 1, which proves the existence of far-field at the aperture. This shows that the employment of slits is not suitable for sensing application due to the absence of the evanescence field. In comparison, the value of $F_{N}$ was greater by using a single slit

As the aperture was replaced with pinholes, the values of $F_{N}$ showed remarkable results where $F_{N}>>1$ as illustrated in Fig. 6. This phenomenon exhibits the presence of near-field and the propagation of evanescent waves at the aperture's end. Value of $F_{N}$ increased in an exponential behaviour as the distance $x$ between lens and aperture was increased. It was also observed that the greater the pinhole's diameter, the smaller the value of $F_{N}$. The stronger optical signal due to the smaller value of $F_{N}$ can be obtained by decreasing the distance $x$. In this work, $5 \mathrm{~cm}$ is observed as the optimum distance $x$ for the enhancement of near-field strength. In comparison between these three pinhole sizes, a $0.8 \mathrm{~mm}$ pinhole results maximum value of $F_{N}$, this yielded the strong near-field. The relationship between $F_{N}$ and distance between lens and aperture, $x$ is shown in Equation 2;

$$
F_{N}=85.999 e^{0.0178 x}
$$

Significant differences in the field intensities, $I$ was observed as different structures of aperture were introduced into the setup as displayed in Figure 7. The appointment of slits aperture, which results in the formation of far-field shows linear enhancement of field intensities with the increment of distance $x$. On the contrary, the same dependent variable displayed opposite behaviour as the slits were replaced with pinholes. This result explains a unique characteristic between the far-field and near-field based on their field intensities' characteristics.

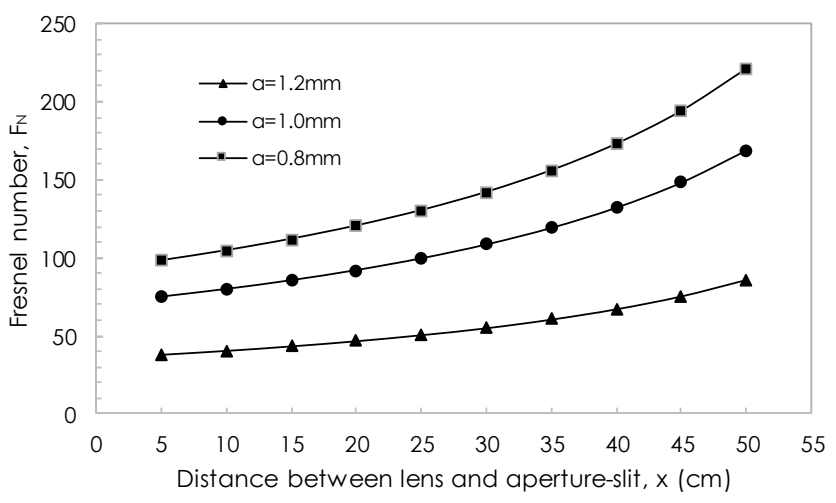

Figure 6. Near-field $\left(F_{N}>>1\right)$ was created as the pinhole apertures were located after the light source double slits. Although the distance between lens and aperture did not give a significant impact on the presence of near-field or farfield, this parameter still influences the value of $F_{N}$. The greater the distance $\mathrm{x}$, the larger the value of $F_{N}$ 


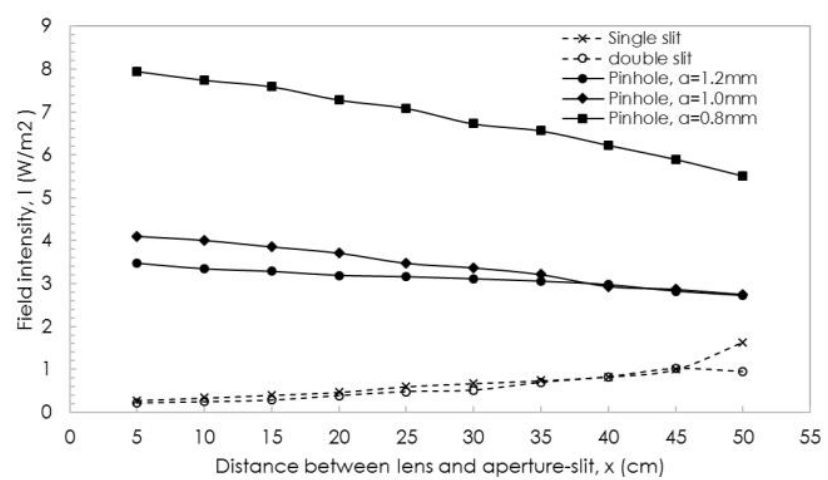

Figure 7. Opposite behaviour of field intensity as light propagated through the slits and pinholes. The field intensity increased with the increment of $\mathrm{x}$ as slits apertures were employed and vice versa as the slits aperture was replaced with a pinhole aperture

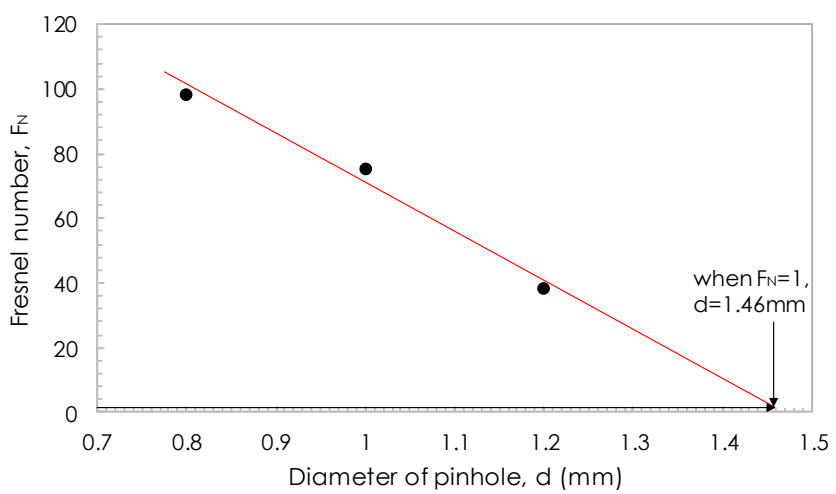

Figure 8. A boundary between near-field and far-field using extrapolation method shows that the maximum diameter of pinhole $\mathrm{a}>>1.46 \mathrm{~mm}$ able to produce near-field when $\mathrm{F}_{\mathrm{N}}=1$

To investigate the influence of pinhole's size on the value of $F_{N}$, we decided to focus at the optimum distance of $x=5 \mathrm{~cm}$, which results in excellent field intensity. At this respective distance, the values of $F_{N}$ were obtained as 38.30, 75.06 and 98.03 as the pinhole's diameters were fixed at $1.2 \mathrm{~mm}$, 1.0mm and $0.8 \mathrm{~mm}$ accordingly as depicted in Fig. 8 . A linear relationship between $F_{N}$ and pinhole's diameters is expressed in Equation 3;

$$
F_{N}=-149.32 x+219.79
$$

By using an extrapolating method, it was found that the maximum diameter of a pinhole for the formation of nearfield can be obtained by employing a pinhole with a diameter smaller than $1.46 \mathrm{~mm}(\mathrm{a}<1.46 \mathrm{~mm})$. This analysis shows that the optical sensing properties due to the presence of evanescence wave able to be produced by manipulating the size of the pinhole aperture.

\section{CONCLUSION}

In this work, the identification of near-field and far-field through assorted sizes of optical apertures has been investigated. The discovery of near-field along the optical path by using pinhole with a diameter smaller than $1.46 \mathrm{~mm}$ reveals the potential of this region to be employed as a sensing area due to the existence of strong evanescence field.

\section{ACKNOWLEDGMENT}

The work was supported by Universiti Sains Islam Malaysia (USIM) and Malaysia Ministry of Higher Education (MOHE) under grant USIM/FRGS/FST/32/51514.

\section{REFERENCES}

[1] Szymanski, C, Wu, C, Hooper, J, Salazar, MA, Perdomo, A, Dukes, A \& McNeill, J 2005, 'Single molecule nanoparticles of the conjugated polymer MEH- PPV, preparation and far-field by near-field scanning optical microscopy', The Journal of Physical Chemistry B, vol. 109, no. 18, pp. 85438546.
[2] Kawata, S, Inouye, Y \& Verma, P 2009, 'Plasmonics for near-field nano-imaging and superlensing', Nature Photonics, vol. 3, no. 7, pp. 388-394.

[3] Mukhtar, WM, Menon, PS \& Shaari, S 2012, 'Effect of taper angle of the optical fiber microprobe in power collection', Advanced Materials Research, vol. 403, pp. 3387-3391.

[4] Zhang, J, Chen, S, Gong, T, Zhang, X \& Zhu, Y 2016, 
'Tapered Fiber Probe Modified by $\mathrm{Ag}$ Nanoparticles for SERS Detection', Plasmonics, vol. 11, no. 3 , pp. 743-751.

[5] Urrutia, A, Bojan, K, Marques, L, Mullaney, K, Goicoechea, J, James, S, Clark, M, Tatam, R \& Korposh, S 2016, 'Novel highly sensitive protein sensors based on tapered optical fibres modified with Au-based nanocoatings', Journal of Sensors, pp. 1-11.

[6] Kharat, HJ, Kakde, KP, Shirale, DJ, Gade, VK, Gaikwad, PD, Savale, PA \& Shirsat, MD 2006, 'Designing of optical fiber sensing probe', Fiber and integrated optics, vol. 25, no. 6, pp. 411-422.

[7] Huo, X, Pan, S \& Wu, S 2006, 'Fabrication of optical fiber probe nano-tips by heated micropulling combined with static chemical etching', in 1st IEEE International Conference on Nano/Micro Engineered and Molecular Systems (NEMS'06), Zhuhai, pp. 254-257.

[8] Xie, X, Chen, Y, Yang, K \& Zhou, J 2014, 'Harnessing the point-spread function for highresolution far-field optical microscopy', Physical Review Letter, vol. 113, no. 26. doi: 10.1103/PhysRevLett.113.263901.

[9] Novotny, L \& Hecht, B 2006, Principles of NanoOptics, Cambridge University Press, United States (US).

[10] Mukhtar, WM, Shaari, S \& Menon, PS 2010, 'Fabrication of optical fiber microprobe using electric arc heating and one-sided pulling technique', in 2010 IEEE Student Conference on Research and Development (SCOReD), Putrajaya, pp. 104-106.

[11] Harun, SW, Jasim, AA, Rahman, HA, Muhammad, MZ \& Ahmad, H 2013, 'Micro-ball lensed fiberbased glucose sensor', IEEE Sensors Journal, vol. 13, no. 1, pp. 348-350.

[12] Halip, NH, Isa, NS, Latif, AA, Mahdi, MA \& Abu, MH 2016, 'Asymmetric fiber taper for narrow linewidth comb filter', Jurnal Teknologi, vol. 78, no. 3 , pp. 117-121.

[13] Musa, B, Kamil, YM, Hafiz, M, Bakar, A, Noor, AS M, Ismail, A \& Mahdi, MA 2016, 'Investigating the effect of taper length on sensitivity of the taperedfiber based temperature sensor', Jurnal Teknologi, vol. 78 , no. 3 , pp. 135-140.

[14] Chen, Y \& Shen, R 2016, 'A reconsideration for forming mechanism of optic fiber probe fabricated by static chemical etching', Applied Physics A, vol. 122, no. 7, pp. 1-5.

[15] Cao, J, Zhao, D \& Mao, Q 2017, 'A highly reproducible and sensitive fiber SERS probe fabricated by direct synthesis of closely packed AgNPs on the silanized fiber taper', Analyst, vol. 142, no. 4, pp. 596-602.

[16] Mukhtar, WM, Menon, PS \& Shaari, S 2012, 'Microfabricated fiber probe by combination of electric arc discharge and chemical etching techniques', Advanced Materials Research, vol. 462, pp. 38-41.

[17] Chen, Z, Dai, Z, Chen, N, Liu, S, Pang, F, Lu, B \& Wang, T 2014, 'Gold nanoparticles-modified tapered fiber nanoprobe for remote SERS detection', IEEE Photonics Technology Letters, vol. 26, no. 8, pp. 777-780.

[18] Hecht, B, Sick, B, Wild, UP, Deckert, V, Zenobi, R, Martin, OJ \& Pohl, DW 2000, 'Scanning near-field optical microscopy with aperture probes: Fundamentals and applications', The Journal of Chemical Physics, vol. 112, no. 18, pp. 7761-7774.

[19] Mukhtar, WM, Shaari, S, Menon, PS \& Ehsan, AA 2012, 'Analysis of biconical taper geometries to the transmission losses in optical microfibers', Optoelectronics and Advanced Materials Rapid Communications, vol. 6, no. 11-12, pp. 988-992.

[20] Tao, M, Wang, A, Xu, L, Jin, Y, Cao, M \& Gu, N 2016, 'Selective electroless silver plating of optical fiber probes with protruding tips', Microsystem Technologies, vol. 22, no. 10, pp. 2487-2491.

[21] Yu, Z, Jin, L, Sun, L, Li, JM, Ran, Y \& Guan, BO 2016, 'Highly sensitive fiber taper interferometric hydrogen sensors', IEEE Photonics Journal, vol. 8, no. 1, pp. 1-9.

[22] D Namenko, D Cassese, M Lazzarino and A Bek, 'Tip-Assisted Optical Nanoscopy for SingleMolecule Activation and Detection', in Novel 
Approaches for Single Molecule Activation and Detection, Springer, Berlin, Heidelberg, 2014, pp. 61-83.

[23] Eleftheriades, GV \& Wong, AMH 2008, 'Holography-inspired screens for sub-wavelength focusing in the near field', IEEE Microwave and Wireless Components Letters, vol. 18, no. 4, pp. 236-238.

[24] Ton, XA, Acha, V, Bonomi, P, Bui, BTS \& Haupt, K 2015, 'A disposable evanescent wave fiber optic sensor coated with a molecularly imprinted polymer as a selective fluorescence probe', Biosensors and Bioelectronics, vol. 64, pp. 359366.

[25] Maliakal, A, Reith, L \& Cabot, S 2016, 'Chemical detection demonstrated using an evanescent wave graphene optical sensor', Applied Physics Letters, vol. 108, no. 15, 153109.

[26] Queiros, RB, Gouveia, C, Fernandes, JRA \& Jorge, PAS 2014, 'Evanescent wave DNA-aptamer biosensor based on long period gratings for the specific recognition of $\mathrm{E}$. coli outer membrane proteins', Biosensors and Bioelectronics, vol. 62, pp. 227-233.

[27] Saleh, BEA \& Teich, MC 1991, Fundamentals of Photonics, John Wiley \& Sons Inc., United States (US).

[28] Mukhtar, WM, Menon, PS \& Shaari, S 2012, 'Study of Fraunhofer diffraction pattern from a circular aperture using an optical fiber microprobe', Advanced Materials Research, vol. 378, pp. 565568.

[29] Kawata, S 2001, Near-field microscope probes utilizing surface plasmon polaritons, eds Kawata S., in Near-Field Optics and Surface Plasmon Polaritons., vol. 81, Springer, Berlin, Heidelberg. 